\title{
Innovation as a Driver of economic growth: An empirical Comparison between China and India
}

\section{Md. Gulam Mokta Dhir ${ }^{1}$, Fatma Zaarour ${ }^{2}$, Md. Emran Uddin ${ }^{3}$, Sanzida Afrose ${ }^{3}$, Mostofa Shamim Ferdous ${ }^{* 4}$}

${ }^{1}$ Dept. of Business Administration, Metropolitan University, Sylhet, Bangladesh

${ }^{2}$ Higher Institute of Management, University of Sousse, Sousse, Tunisia

${ }^{3}$ Sustainable Development Management, Rhein-Waal University, Germany

${ }^{4}$ Research Associate, Dept. of Business Administration, Metropolitan University, Sylhet, Bangladesh

*Corresponding Author Email: shamim.active@gmail.com

Received: 16.11.2021; Revised: 21.12.2021; Accepted:28.12.2021

OSociety for Himalayan Action Research and Development

\begin{abstract}
This study focuses on the relationship between patent application and economic development in two most important global economies China and India. We have used time series data and have applied ARDL bound testing approach for finding out a long run relationship. The outputs show that there must be a long run relationship between patent registration and economic development. We can conclude out of this those innovations (patent) might be a very vital factor for economic development in China and India. It has also helped us to draw some policies' recommendations.
\end{abstract}

Keywords: China, India, Economic growth, Patent application, ARDL, Bound test

\section{Introduction}

Intellectual property plays an important role in assessing and simulating creativity and inventions wit growing knowledge in the modern economy. It also has the potential to influence a country's GDP growth. The percentage increase in GDP is invariably determined by the patenting of new inventions. A patent is a legal right that grants applicants (or inventors) exclusive rights to their inventions for a set period of time (generally 20 years). As GDP and GDP per capita are two important parameters of macroeconomic performance of a country, some studies have examined and analysed the relationship between patents and the other ones. The goal of this paper is to conduct an empirical research on the relationship between number of patent registrations and economic growth. Schumpeter (1947), like other economists, emphasizes the importance of entrepreneurship and innovation in the process of economic growth. Schumpeter (op cit) demonstrated in "Theoretical Problems of Economic Growth" that scholars consider various factors that enhance economic growth: physical environment, social organization, institutions, technology, and so on. Creative response is important in the innovation process. In the economic sphere, creative response simply means combining existing productive resources in new ways 
or for new purposes, and because this function defines the economic type known as the entrepreneur, we can reformulate the preceding suggestions by saying that we should recognize the importance of, and systematically investigate, entrepreneurship as a factor of economic growth. Entrepreneurship with innovation significantly depends on patent registration to protect the innovation which eventually can be a good indicator of economic growth. Let us see what the study reveals after analysing the variables.

The remainder of the paper is structured as follows. Section II provides a synopsis of the literature. Section III discusses the paper's econometric methodology. Section V contains the empirical results of tests, and Section VI contains some conclusions

\section{Literature Review}

Patents exist to encourage inventive activity and to facilitate the incorporation of new technologies into the economy as a whole. One mechanism for technology diffusion in an economy is the patent system. Patent is the only recognized way to protect innovation and technology. Technology reaches on its peak if it ensures innovation. An innovation system is made up of individuals and organizations that invest time, energy, and resources in the creation of scientific and technical knowledge, both directly and indirectly (Katz, 2006). Technological change is now regarded as a major source of long-run productivity growth, and innovation is no longer regarded as an exogenous process (Josheski, Koteski 2011). Patents have grown in importance, particularly in the last two decades and innovations are protected by patent. The growth of patent has a positive relationship with growth of GDP. Gerguri (2010) have highlighted several core conditions that enable innovation and encourage economic growth: strong intellectual property standards and effective enforcement, vigorous competition and contestable markets, a strong and sustainable fundamental research and development infrastructure, encouraging information and technology communication developments, a strong emphasis on education at all levels, and so on. National patents provide insight into the extent to which a regional economy is developing and commercializing new-to-the-country technologies (Guan \& Liu, 2005). National patents are divided into three categories: invention patents, utility patents, and design patents. Chen and Puttitanun, (2005) and Briggs (2007) who have investigated the question of whether the patent has any contribution to GDP using theoretical models and econometric analyses of multi-country data sets have identified a U-shaped relationship between GDP and the Ginarte-Park Index of patent rights. Maskus (2000) first openly acknowledged the U-shaped association using estimated coefficients for log GNP per capita and on the instrument-corrected Rapp-Rozek Patent Index in a cross-section regression. A study conducted by Sinha (2007) on Japan and South Korea on the relationship between patent and economic growth found a 
positive cointegration between the variables. She also found two way causality between GDP growth with growth of the number of patent. Roman Gurbiel (2002) from his study on technology and innovation based on EU countries concludes that in today's global economy, innovation and technology transfer are the primary drivers of economic growth. He discovered a strong relationship between the intensity of technology transfer and a country's ability to innovate. Recently Hasan \& Tucci (2010) and Kim et al.(2012) By addressing some new questions, the research line was extended in an attempt to link innovation to economic growth. These researchers investigated the importance of both the quantity and quality of innovation in economic growth using global patent data from 58 countries. Furthermore, this study found that countries with higher quality patents have higher economic growth. On the side of the coin Fleisher et al. (2013), in line with Kim et al.(2012), have discovered that between 1992 and 2002, patent laws, including China's adoption of Trade Related Aspects of Intellectual Property Rights standards, had no direct effect on China's productivity. Some other researchers have also some orthodox statement instead of general findings of positive relationship between patent and economic growth. Briggs (2007) argues that The U-shaped relationship found in cross-section and panel regression estimates between GDP and patent strength is a misleading guide to the longitudinal experiences of the vast majority of countries in the Ginarte-Park data set. In the study Changtao Wang (2013) Patent and trademark statistics are used in the study conducted by Changtao Wang (2013) as innovation proxies to examine the long-run relationship between innovation and output in countries with a long-established intellectual property rights system. The findings suggest that innovation may no longer be a positive force in driving economic growth.

\section{Methodology}

Here we have used the mixed stationarity of our variables that allows the application of ARDL bounds testing approach that has been developed by Pesaran et al (2001). The basic issue of ARDL model is that it can be used irrespective of the order of stationarity of the variables, though there is an exception for I(2) variables. We can specify the ARDL model can be specified in the following way (Pesaran et al op cit):

$\Delta G D P_{t}=\alpha_{0}+\alpha_{1} G D P_{t-1}+\alpha_{2}$ Patent $_{t-1}+\sum_{i=1}^{n} \varphi_{i} \Delta G D P_{t-i}++\sum_{i=1}^{n} \pi_{i} \Delta$ Patent $_{t-i}+\mu_{t}$

Where $\Delta$ is the differenced operator, $\alpha 1 ; \alpha 2$ are the long run estimates, $\varphi_{i}, \pi_{i}, \mathrm{i}$ are short-run estimates, and $\mu_{\mathrm{t}}$ is residual term in time $\mathrm{t}$.

The ARDL may choose its appropriate lag length in automatic way. The appropriate calculation of the Fstatistic relies on the proper lag order selection of the series integrated in the model. The asymptotic 
critical values produced by Pesaran et al. (op cit) are compared with the F-statistic to find the co integration. The condition for co integration is that the F-statistics be greater than the upper critical values - otherwise means absence of cointegration. If cointegration is recognized, then we go on to error correction model (ECM) following Pesaran et al. with the short-run dynamics symbolized as:

$\Delta G D P_{t}=\alpha_{1}+\sum_{i=1}^{n} \varphi_{i} \Delta G D P_{t-i}++\sum_{i=1}^{n} \pi_{i} \Delta$ Patent $_{t-i}+$ recm $_{t-1}+\mu_{t}$

The short-run coefficients are as well $\varphi_{i}, \pi_{i}$; ,i, while $\gamma$ is the speed of adjustment coefficient to equilibrium.

\section{Empirical Analysis}

Before estimating Auto Regressive Distributed Lag model, we need to check for stationarity of the variables via Augmented Dickey-Fuller Test and Philips-Perron Test for being sure that none of the variables is integrated of second order. Because bound testing procedure gets broken down (Pesaran et al op cit ). The output of unit root tests namely ADF and PP Test has been presented in Table 1 and 2. The results show that the variables are stationary in level and first difference that means $\mathrm{I}(0), \mathrm{I}(1)$ or both. The level of significance is $5 \%$. The interesting matter is that the most macroeconomic variables have the propensities of being stationary at level that means $\mathrm{I}(0)$, if not it usually gets stationary at first difference, I(1). That's why ARDL method usually does not need pre-testing for the unit root. Moreover, this method considers enough lags of the variables that serve as a proper instrument to remove the endogeneity issue (Bahmani-Oskooee \& Hajilee, 2010). Pesaran et al. (2001) describe this instrument as an approach of general method, because it provides very flexible choice for the dynamic lag structure and also allow for short run feedbacks.

The long run and short run test results presented in Table 3, Table 4 show that F value is 3.532259 For China, 9.543696 For India. For India there must be a co-integration relationship at 5\% level of significance as the $\mathrm{F}$ value lie above upper bound critical value at 5\%. For China, it's inconclusive at 10\% level of significance. Though for the presence of co-integration relationship, the co-efficient of ECM term should be statistically significant. Apart from that it must lie between -1 to 0 . It will help to check and test the speed of adjustment back to equilibrium after a shock occurs (Banerjee et al., 1998). The ECM terms presented in Tables 5 and 6 prove that there must be long-run relationship between dependent and explanatory variables as the ECM terms are statistically significant. 
Himalayan J. Soc. Sci. \& Humanities ISSN: 0975-9891

Vol. 16, (2021) 1-12

DOI: https://doi.org/10.51220/hjssh.v16i1.1

Table 1: Unit Root Tests (PP)

\begin{tabular}{|c|c|c|c|c|c|}
\hline \multirow{2}{*}{\multicolumn{6}{|c|}{ UNIT ROOT TEST TABLE (PP) }} \\
\hline \multicolumn{4}{|c|}{ At Level } & & \\
\hline & & GDP_CN & GDP_IN & PATENT_CN_GROWTH & PATENT_IN_GROWTH \\
\hline \multirow{3}{*}{$\begin{array}{l}\text { With } \\
\text { Constant }\end{array}$} & $\mathrm{t}$-Statistic & -2.9822 & -5.0642 & -4.3109 & -5.2821 \\
\hline & Prob. & 0.047 & 0.0002 & 0.0018 & 0.0001 \\
\hline & & ** & $* * *$ & $* * *$ & $* * *$ \\
\hline \multirow{3}{*}{$\begin{array}{l}\text { With } \\
\text { Constant } \\
\text { \& Trend }\end{array}$} & t-Statistic & -2.9938 & -11.6974 & -4.0538 & -5.5499 \\
\hline & Prob. & 0.149 & 0 & 0.0164 & 0.0004 \\
\hline & & n0 & $* * *$ & $* *$ & $* * *$ \\
\hline \multirow{3}{*}{$\begin{array}{l}\text { Without } \\
\text { Constant } \\
\text { \& Trend }\end{array}$} & $\mathrm{t}$-Statistic & -1.2307 & -0.9788 & -2.1322 & -2.6171 \\
\hline & Prob. & 0.196 & 0.2866 & 0.0336 & 0.0105 \\
\hline & & n0 & n0 & $* *$ & $* *$ \\
\hline \multicolumn{6}{|c|}{ At First Difference } \\
\hline & & $\begin{array}{l}\text { d(GDP_CN } \\
\text { ) }\end{array}$ & $\begin{array}{l}\mathrm{d}\left(\mathrm{GDP} \_I N\right. \\
)\end{array}$ & $\begin{array}{l}\text { d(PATENT_CN_GROWTH } \\
\text { ) }\end{array}$ & $\begin{array}{l}\text { d(PATENT_IN_GROWTH } \\
\text { ) }\end{array}$ \\
\hline \multirow{3}{*}{$\begin{array}{l}\text { With } \\
\text { Constant }\end{array}$} & t-Statistic & -8.0411 & -21.1871 & -10.0224 & -22.5046 \\
\hline & Prob. & 0 & 0.0001 & 0 & 0.0001 \\
\hline & & $* * *$ & $* * *$ & $* * *$ & $* * *$ \\
\hline \multirow{3}{*}{$\begin{array}{l}\text { With } \\
\text { Constant } \\
\text { \& Trend }\end{array}$} & t-Statistic & -9.2204 & -21.1174 & -11.3524 & -24.6609 \\
\hline & Prob. & 0 & 0 & 0 & 0 \\
\hline & & $* * *$ & **** & $* * *$ & $* * *$ \\
\hline \multirow{3}{*}{$\begin{array}{l}\text { Without } \\
\text { Constant } \\
\text { \& Trend }\end{array}$} & $\mathrm{t}$-Statistic & -8.1132 & -19.8285 & -10.2223 & -17.6062 \\
\hline & Prob. & 0 & 0 & 0 & 0 \\
\hline & & $* * *$ & $* * *$ & $* * *$ & $* * *$ \\
\hline
\end{tabular}

Table 2: Unit Root Tests (ADF)

\begin{tabular}{|c|c|c|c|c|c|}
\hline \multicolumn{6}{|l|}{ At Level } \\
\hline & & GDP_CN & GDP_IN & PATENT_CN_GROWTH & PATENT_IN_GROWTH \\
\hline \multirow{3}{*}{$\begin{array}{l}\text { With } \\
\text { Constant }\end{array}$} & t-Statistic & -2.9454 & -5.0947 & -4.2949 & -5.2821 \\
\hline & Prob. & 0.0509 & 0.0002 & 0.0019 & 0.0001 \\
\hline & & * & $* * *$ & $* * *$ & $* * *$ \\
\hline \multirow{3}{*}{$\begin{array}{l}\text { With } \\
\text { Constant } \\
\text { \& Trend }\end{array}$} & t-Statistic & -2.963 & -5.5283 & -4.0337 & -5.5499 \\
\hline & Prob. & 0.1573 & 0.0004 & 0.0172 & 0.0004 \\
\hline & & n0 & $* * *$ & $* *$ & $* * *$ \\
\hline \multirow{3}{*}{$\begin{array}{l}\text { Without } \\
\text { Constant } \\
\& \text { Trend }\end{array}$} & $\mathrm{t}$-Statistic & -1.1847 & -1.0542 & -1.4073 & -0.7789 \\
\hline & Prob. & 0.211 & 0.2574 & 0.1453 & 0.3705 \\
\hline & & n0 & n0 & n0 & n0 \\
\hline
\end{tabular}


Himalayan J. Soc. Sci. \& Humanities ISSN: 0975-9891

Vol. 16, (2021) 1-12

DOI: https://doi.org/10.51220/hjssh.v16i1.1

\begin{tabular}{|c|c|c|c|c|c|}
\hline & & $\begin{array}{l}\mathrm{d}\left(\mathrm{GDP} \_\mathrm{CN}\right. \\
{ }^{2}\end{array}$ & $\begin{array}{l}\mathrm{d}\left(\mathrm{GDP} \_\mathrm{IN}\right. \\
\end{array}$ & $\begin{array}{l}\text { d(PATENT_CN_GROWTH } \\
\text { ) }\end{array}$ & $\begin{array}{l}\text { d(PATENT_IN_GROWTH } \\
\text { ) }\end{array}$ \\
\hline \multirow{3}{*}{$\begin{array}{l}\text { With } \\
\text { Constant }\end{array}$} & $\mathrm{t}$-Statistic & -5.1795 & -5.783 & -8.5209 & -7.5953 \\
\hline & Prob. & 0.0002 & 0 & 0 & 0 \\
\hline & & $* * *$ & $* * *$ & $* * *$ & $* * *$ \\
\hline \multirow{3}{*}{$\begin{array}{l}\text { With } \\
\text { Constant } \\
\text { \& Trend }\end{array}$} & t-Statistic & -5.0955 & -5.6883 & -8.5818 & -7.4561 \\
\hline & Prob. & 0.0013 & 0.0003 & 0 & 0 \\
\hline & & $* * *$ & $* * *$ & $* * *$ & $* * *$ \\
\hline \multirow{3}{*}{$\begin{array}{l}\text { Without } \\
\text { Constant } \\
\text { \& Trend }\end{array}$} & t-Statistic & -5.2738 & -5.8657 & -6.4852 & -7.6848 \\
\hline & Prob. & 0 & 0 & 0 & 0 \\
\hline & & $* * *$ & $* * *$ & $* * *$ & $* * *$ \\
\hline
\end{tabular}

Notes: $(*)$ Significant at the $10 \% ;(* *)$ Significant at the 5\%; $(* * *)$ Significant at the $1 \%$. and (no) Not Significant

*MacKinnon (1996) one-sided p-values.

Table 3: ARDL Long Run Form, Short Run Form and Bound Test - India

\begin{tabular}{|c|c|c|c|c|c|}
\hline \multicolumn{6}{|c|}{ ARDL Long Run Form and Bounds Test } \\
\hline \multicolumn{6}{|c|}{ Dependent Variable: D(GDP_IN) } \\
\hline \multicolumn{6}{|c|}{ Selected Model: $\operatorname{ARDL}(1,3)$} \\
\hline \multicolumn{6}{|c|}{ Case 2: Restricted Constant and No Trend } \\
\hline \multicolumn{6}{|c|}{ Conditional Error Correction Regression } \\
\hline \multicolumn{2}{|l|}{ Variable } & Coefficient & Std. Error & t-Statistic & Prob. \\
\hline \multicolumn{2}{|l|}{$\mathrm{C}$} & 5.481391 & 1.342056 & 4.084322 & 0.0004 \\
\hline \multicolumn{2}{|l|}{ GDP_IN(-1)* } & -1.0299 & 0.192785 & -5.34222 & 0 \\
\hline \multicolumn{2}{|c|}{ PATENT_IN_GROWTH(-1) } & 12.04175 & 8.442113 & 1.42639 & 0.1661 \\
\hline \multicolumn{2}{|c|}{ D(PATENT_IN_GROWTH) } & -2.61656 & 4.131114 & -0.63338 & 0.5322 \\
\hline \multicolumn{2}{|c|}{ D(PATENT_IN_GROWTH(-1)) } & -9.66409 & 5.84826 & -1.65247 & 0.1109 \\
\hline \multicolumn{2}{|c|}{ D(PATENT_IN_GROWTH(-2)) } & -4.929 & 4.158983 & -1.18515 & 0.2471 \\
\hline \multicolumn{6}{|c|}{ * p-value incompatible with t-Bounds distribution. } \\
\hline \multicolumn{6}{|l|}{ Levels Equation } \\
\hline \multicolumn{6}{|c|}{ Case 2: Restricted Constant and No Trend } \\
\hline \multicolumn{2}{|l|}{ Variable } & Coefficient & $\begin{array}{l}\text { Std. } \\
\text { Error }\end{array}$ & $\begin{array}{l}\mathrm{t}- \\
\text { Statistic }\end{array}$ & Prob. \\
\hline \multicolumn{2}{|c|}{ PATENT_IN_GROWTH } & 11.69212 & 7.94799 & 1.471079 & 0.1537 \\
\hline \multicolumn{2}{|l|}{$\mathrm{C}$} & 5.322242 & 0.840873 & 6.329423 & 0 \\
\hline \multicolumn{6}{|c|}{$\begin{array}{l}\text { EC }=\text { GDP_IN - (11.6921*PATENT_IN_GROWTH + } \\
5.3222)\end{array}$} \\
\hline F-Bounds Test & $\begin{array}{l}\text { Null Hypothesis: No levels } \\
\text { relationship }\end{array}$ & & & & \\
\hline \multirow[t]{2}{*}{ Test Statistic } & Value & Signif. & \multicolumn{2}{|l|}{$\mathrm{I}(0)$} & $\mathrm{I}(1)$ \\
\hline & & & \multicolumn{2}{|c|}{ Asymptotic: $n=1000$} & \\
\hline F-statistic & 9.543696 & $10 \%$ & 3.02 & & 3.51 \\
\hline
\end{tabular}


Himalayan J. Soc. Sci. \& Humanities ISSN: 0975-9891

Vol. 16, (2021) 1-12

DOI: https://doi.org/10.51220/hjssh.v16i1.1

\begin{tabular}{|c|c|c|c|c|}
\hline \multirow[t]{3}{*}{$\mathrm{k}$} & 1 & $5 \%$ & 3.62 & 4.16 \\
\hline & & $2.50 \%$ & 4.18 & 4.79 \\
\hline & & $1 \%$ & 4.94 & 5.58 \\
\hline \multirow[t]{8}{*}{ Actual Sample Size } & 31 & & Finite Sample: $n=35$ & \\
\hline & & $10 \%$ & 3.223 & 3.757 \\
\hline & & $5 \%$ & 3.957 & 4.53 \\
\hline & & $1 \%$ & 5.763 & 6.48 \\
\hline & & & Finite Sample: $n=30$ & \\
\hline & & $10 \%$ & 3.303 & 3.797 \\
\hline & & $5 \%$ & 4.09 & 4.663 \\
\hline & & $1 \%$ & 6.027 & 6.76 \\
\hline
\end{tabular}

Table 4: ARDL Long Run Form, Short Run Form and Bound Test - China

\begin{tabular}{|l|l|l|l|l|}
\hline ARDL Long Run Form and Bounds Test & \multicolumn{2}{l|}{} & \\
\hline Dependent Variable: D(GDP_CN) & \multicolumn{5}{l|}{} \\
\hline Selected Model: ARDL(4,1) & \multicolumn{5}{l|}{} \\
\hline Case 2: Restricted Constant and No Trend & & & \\
\hline Conditional Error Correction Regression & \multicolumn{5}{l|}{} \\
\hline Variable & Coefficient & Std. Error & t-Statistic & Prob. \\
\hline C & 4.001648 & 1.983862 & 2.0171 & 0.0555 \\
\hline GDP_CN(-1)* & -0.51952 & 0.190562 & -2.72626 & 0.012 \\
\hline PATENT_CN_GROWTH(-1) & 4.270561 & 2.715155 & 1.572861 & 0.1294 \\
\hline D(GDP_CN(-1)) & 0.686521 & 0.200794 & 3.41903 & 0.0023 \\
\hline D(GDP_CN(-2)) & -0.19259 & 0.183745 & -1.04813 & 0.3055 \\
\hline D(GDP_CN(-3)) & 0.387609 & 0.162053 & 2.391866 & 0.0253 \\
\hline D(PATENT_CN_GROWTH) & 1.195058 & 2.17095 & 0.550477 & 0.5873 \\
\hline
\end{tabular}

* p-value incompatible with t-Bounds distribution.

Levels Equation

Case 2: Restricted Constant and No Trend

\begin{tabular}{|l|l|l|l|l|}
\hline Variable & Coefficient & $\begin{array}{l}\text { Std. } \\
\text { Error }\end{array}$ & $\begin{array}{l}\text { t- } \\
\text { Statistic }\end{array}$ & Prob. \\
\hline PATENT_CN_GROWTH & 8.220159 & 6.191875 & 1.327572 & 0.1973 \\
\hline C & 7.702544 & 1.475325 & 5.220915 & 0 \\
\hline $\begin{array}{l}\text { EC }=\text { GDP_CN - (8.2202*PATENT_CN_GROWTH + } \\
7.7025)\end{array}$ & & & & \\
\hline
\end{tabular}

\begin{tabular}{|l|l|l|l|l|}
\hline & $\begin{array}{l}\text { Null } \\
\text { Hypothesis: } \\
\text { No levels } \\
\text { relationship }\end{array}$ & & & \\
\hline Test Statistic & Value & Signif. & $\mathrm{I}(0)$ & $\mathrm{I}(1)$ \\
\hline
\end{tabular}


Himalayan J. Soc. Sci. \& Humanities ISSN: 0975-9891

Vol. 16, (2021) 1-12

DOI: https://doi.org/10.51220/hjssh.v16i1.1

\begin{tabular}{|c|c|c|c|c|}
\hline & & & $\begin{array}{l}\text { Asymptotic: } \\
\mathrm{n}=1000\end{array}$ & \\
\hline F-statistic & 3.532259 & $10 \%$ & 3.02 & 3.51 \\
\hline \multirow[t]{3}{*}{$\mathrm{k}$} & 1 & $5 \%$ & 3.62 & 4.16 \\
\hline & & $2.50 \%$ & 4.18 & 4.79 \\
\hline & & $1 \%$ & 4.94 & 5.58 \\
\hline \multirow[t]{4}{*}{ Actual Sample Size } & 30 & & $\begin{array}{l}\text { Finite Sample: } \\
\mathrm{n}=30\end{array}$ & \\
\hline & & $10 \%$ & 3.303 & 3.797 \\
\hline & & $5 \%$ & 4.09 & 4.663 \\
\hline & & $1 \%$ & 6.027 & 6.76 \\
\hline
\end{tabular}

Table 5: ARDL Error Correction Regression - India

\begin{tabular}{|c|c|c|c|c|}
\hline \multicolumn{5}{|c|}{ Dependent Variable: D(GDP_IN) } \\
\hline \multicolumn{5}{|l|}{ Selected Model: $\operatorname{ARDL}(1,3)$} \\
\hline \multicolumn{5}{|c|}{ Case 2: Restricted Constant and No Trend } \\
\hline \multicolumn{5}{|l|}{ ECM Regression } \\
\hline Variable & Coefficient & Std. Error & $\mathrm{t}$-Statistic & Prob. \\
\hline D(PATENT_IN_GROWTH) & -2.616561 & 3.458605 & -0.756537 & 0.4564 \\
\hline $\begin{array}{l}\text { D(PATENT_IN_GROWTH(- } \\
\text { 1)) }\end{array}$ & -9.664094 & 4.240806 & -2.278834 & 0.0315 \\
\hline $\begin{array}{l}\text { D(PATENT_IN_GROWTH(- } \\
\text { 2)) }\end{array}$ & -4.929003 & 3.612912 & -1.364274 & 0.1846 \\
\hline CointEq(-1)* & -1.029903 & 0.18521 & -5.560717 & 0 \\
\hline R-squared & 0.564707 & Mean dependent var & & 0.082827 \\
\hline Adjusted R-squared & 0.516342 & S.D. dependent var & & 2.690061 \\
\hline S.E. of regression & 1.870818 & $\begin{array}{c}\text { Akaike } \\
\text { criterion }\end{array} \quad$ info & & 4.210543 \\
\hline Sum squared resid & 94.49893 & Schwarz criterion & & 4.395573 \\
\hline Log likelihood & -61.26341 & Hannan-Quinn criter. & & 4.270858 \\
\hline Durbin-Watson stat & 1.674919 & & & \\
\hline
\end{tabular}

* p-value incompatible with t-Bounds distribution.

Table 6 : ARDL Error Correction Regression - China

\begin{tabular}{|c|c|c|c|c|}
\hline \multicolumn{5}{|c|}{ Dependent Variable: D(GDP_CN) } \\
\hline \multicolumn{5}{|c|}{ Selected Model: $\operatorname{ARDL}(4,1)$} \\
\hline \multicolumn{5}{|l|}{ ECM Regression } \\
\hline \multicolumn{5}{|c|}{ Case 2: Restricted Constant and No Trend } \\
\hline \multicolumn{5}{|l|}{ ECM Regression } \\
\hline Variable & Coefficient & Std. Error & t-Statistic & Prob. \\
\hline $\mathrm{D}\left(\mathrm{GDP} \_\mathrm{CN}(-1)\right)$ & 0.686521 & 0.1815 & 3.782474 & 0.001 \\
\hline $\mathrm{D}\left(\mathrm{GDP} \_\mathrm{CN}(-2)\right)$ & -0.192589 & 0.175259 & -1.098882 & 0.2832 \\
\hline
\end{tabular}


Himalayan J. Soc. Sci. \& Humanities ISSN: 0975-9891

Vol. 16, (2021) 1-12

DOI: https://doi.org/10.51220/hjssh.v16i1.1

\begin{tabular}{|l|l|l|l|l|} 
D(GDP_CN(-3)) & 0.387609 & 0.153101 & 2.531719 & 0.0186 \\
\hline D(PATENT_CN_GROWTH) & 1.195058 & 1.857629 & 0.643325 & 0.5264 \\
\hline CointEq(-1)* & -0.519523 & 0.153078 & -3.393853 & 0.0025 \\
\hline R-squared & 0.573265 & Mean dependent var & & -0.149094 \\
\hline Adjusted R-squared & 0.504987 & S.D. dependent var & & 2.238253 \\
\hline S.E. of regression & 1.574771 & Akaike info criterion & & 3.897109 \\
\hline Sum squared resid & 61.9976 & Schwarz criterion & & 4.130642 \\
\hline Log likelihood & -53.45663 & Hannan-Quinn criter. & & 3.971818 \\
\hline Durbin-Watson stat & 2.154108 & & & \\
\hline
\end{tabular}

$*$ p-value incompatible with t-Bounds distribution.

The long run estimates have been presented in Table 3 and 4. It shows that the coefficient of patent growth Rate is insignificant for China and India. Here the statistical insignificance might have happened because of the assumption of symmetric effect in linear A.R.D.L.

The residual statistics and stability tests' outputs have been showed in the Tables 7-8 and Figs 1-6. They prove that the models are stable, free from serial correlation and rid of heteroskedasticity.

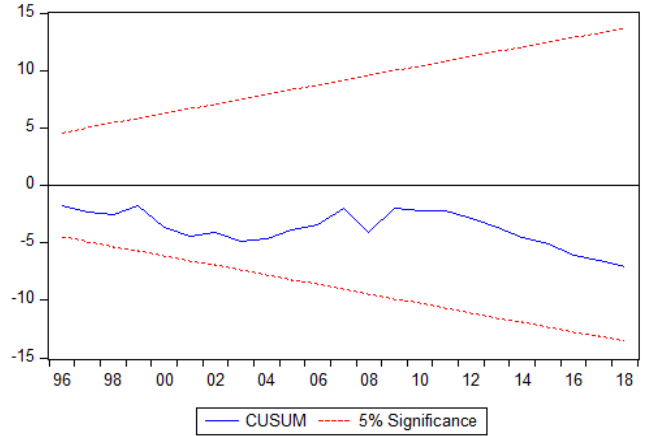

Fig 1: CUSUM-CHINA

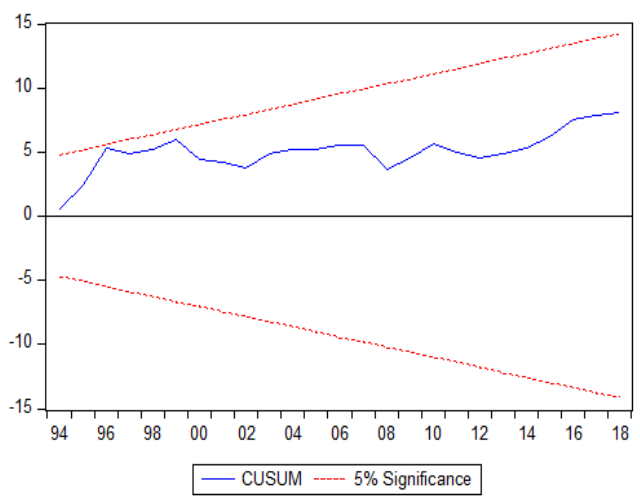

Fig 3: CUSUM-INDIA

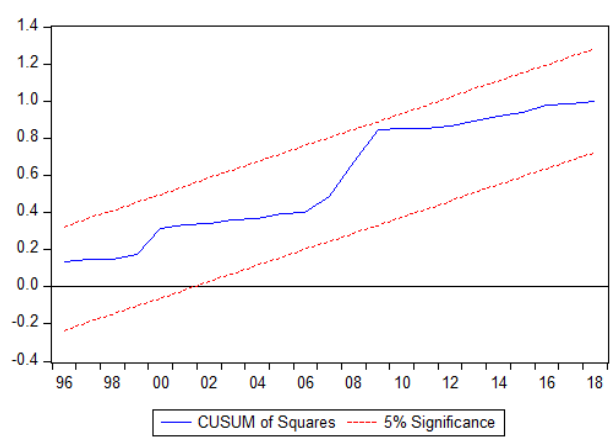

Fig 2: CUSUMSQ-CHINA

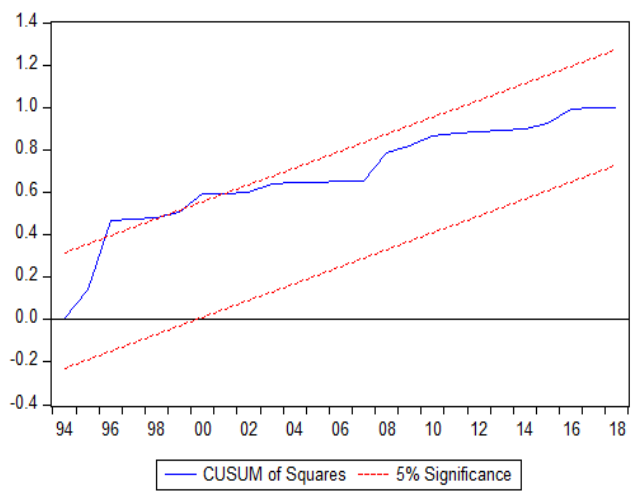

Fig 4: CUSUMSQ-INDIA 
Himalayan J. Soc. Sci. \& Humanities ISSN: 0975-9891

Vol. 16, (2021) 1-12

DOI: https://doi.org/10.51220/hjssh.v16i1.1

Table 7: Breusch-Godfrey Serial Correlation LM Test

\begin{tabular}{|l|l|l|l|l|}
\hline India & Prob. F(2,23) & 0.2505 \\
\hline F-statistic & 1.47118 & Prob. Chi-Square(2) & 0.1724 \\
\hline Obs*R-squared & 3.515993 & & 0.1785 \\
\hline China & 1.806485 & Prob. F(3,20) & 0.0939 \\
\hline F-statistic & 6.396033 & Prob. Chi-Square(3) & \\
\hline Obs*R-squared &
\end{tabular}

Table 8: Heteroskedasticity Test: Breusch-Pagan-Godfrey

\begin{tabular}{|l|l|l|l|l|}
\hline China & 1.393118 & Prob. F(6,23) & 0.2595 \\
\hline F-statistic & 7.996544 & Prob. Chi-Square(6) & 0.2384 \\
\hline Obs*R-squared & 6.335017 & Prob. Chi-Square(6) & 0.3867 \\
\hline Scaled explained SS & 0.776438 & Prob. F(5,25) & 0.5759 \\
\hline India & 4.166856 & Prob. Chi-Square(5) & 0.5257 \\
\hline F-statistic & 4.909492 & Prob. Chi-Square(5) & 0.427 \\
\hline Obs*R-squared &
\end{tabular}

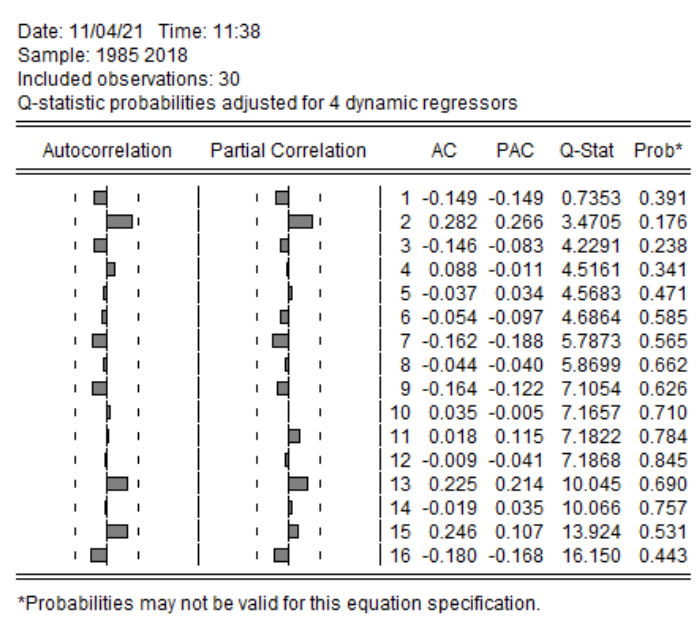

Fig 5: correlogram-CHINA

\begin{tabular}{|c|c|c|c|c|c|c|c|c|}
\hline \multicolumn{2}{|c|}{ Autocorrelation } & \multicolumn{2}{|c|}{ Partial Correlation } & \multirow{2}{*}{\multicolumn{2}{|c|}{$\begin{array}{cc}\text { AC } \\
10.075\end{array}$}} & \multirow{2}{*}{$\begin{array}{l}\text { PAC } \\
0.075\end{array}$} & \multirow{2}{*}{$\begin{array}{l}\text { Q-Stat } \\
0.1920\end{array}$} & \multirow{2}{*}{$\begin{array}{c}\text { Prob* }^{*} \\
0.661\end{array}$} \\
\hline $1 \quad$ & 1 & $1 \mathrm{~b}$ & 1 & & & & & \\
\hline 1 미 & 1 & 1 미 & 1 & 2 & -0.160 & -0.167 & 1.0988 & 0.577 \\
\hline 1믄 & 1 & 1 면 & 1 & 3 & -0.212 & -0.191 & 2.7334 & 0.435 \\
\hline 10 & 1 & 10 & 1 & 4 & -0.070 & -0.073 & 2.9201 & 0.571 \\
\hline 1 d & 1 & 1 단. & 1 & 5 & -0.086 & -0.153 & 3.2123 & 0.667 \\
\hline $1 \quad$ & 1 & 10 & 1 & 6 & 0.173 & 0.131 & 4.4394 & 0.617 \\
\hline 10 & 1 & 11 & 1 & 7 & 0.074 & -0.008 & 4.6714 & 0.700 \\
\hline 10 & 1 & 10 & 1 & 8 & -0.101 & -0.118 & 5.1294 & 0.744 \\
\hline 1 d & 1 & 11 & 1 & 9 & -0.083 & -0.017 & 5.4466 & 0.794 \\
\hline $1 \quad$ & 1 & 10 & 1 & $\mid 10$ & 0.104 & 0.101 & 5.9776 & 0.817 \\
\hline $1 E$ & ال & 1 & ינ & 11 & 0.296 & 0.310 & 10.473 & 0.488 \\
\hline 1므 & 1 & 1口 & 1 & $\mid 12$ & -0.206 & -0.298 & 12.753 & 0.387 \\
\hline 1 미 & 1 & 1 & 1 & 13 & -0.145 & -0.042 & 13.945 & 0.378 \\
\hline 14 & 1 & $1 \quad b$ & 1 & 14 & -0.057 & 0.042 & 14.143 & 0.439 \\
\hline 11 & 1 & 1 & 1 & $\mid 15$ & -0.015 & -0.052 & 14.158 & 0.514 \\
\hline 1 & 1 & 14 & 1 & 16 & -0.032 & -0.070 & 14.226 & 0.582 \\
\hline
\end{tabular}

Fig 6: correlogram-INDIA

\section{Conclusion}

Though this paper explores the relationship between patent application and economic growth, there has been some limitation of this paper. This paper has used only different models and estimation techniques on patent application and growth. For further research we should put light on the R\&D and its multiple 
Himalayan J. Soc. Sci. \& Humanities ISSN: 0975-9891

Vol. 16, (2021) 1-12

DOI: https://doi.org/10.51220/hjssh.v16i1.1

types and its expenditures. We should also classify sector wise expenditures as well to find out sectors' specific innovation and their roles for economic growth. The government should be more effective for promoting and fostering innovation within the economy. On the contrary, just counting higher number of patent applications could not classify between ordinary patents and higher quality of patents. Moreover, an economy should not lower its standards by granting more patents. Government should charge subsidize amount of fess to register patents. For higher level of innovation activities, government should provide motivational measures to foster the environment of innovations.

\section{References:}

Bahmani-Oskooee M, Hajilee M (2010). On the relation between currency depreciation and domestic investment. Journal of Post Keynesian Economics, 32(4), 645-660.

Banerjee A, Dolado J, Mestre R (1998). Error-correction mechanism tests for cointegration in a single-equation framework. Journal of time series analysis, 19(3), 267-283.

Briggs, K N (2007). "Intellectual Property Rights: A Longitudinal vs. Cross-Sectional Study." Unpublished Manuscript, University of North Carolina at Chapel Hill

Chen Y, T Puttitanun (2005). "Intellectual Property Rights and Innovation in Developing Countries", Journal of Development Economics 78(2), pp. 471-493.

Fleisher B M, McGuire W H, Smith A N, Zhou M (2013). Patent law, TRIPS, and economic growth: evidence from China. Asia-Pacific Journal of Accounting \& Economics, 20(1), 4-19.

Gerguri S, (2010) The impact of innovation into the economic growth [Electronic resource]/ S. Gerguri, V. Ramadani // MPRA paper 2010, Ne 22270. Access muenchen.de/22270/1/The_impact_of_innovation_into_economic_growth-final_2008-eng-ShqipeVeland.pdf.

Guan J C, Liu S Z (2005). Comparing regional innovative capacities of PR China-based on data analysis of the national patents. International Journal of

Technology Management, 32, 225-245.

Gurbiel R (2002) Impact of innovation and technology transfer on economic growth: the Central and Eastern Europe experience

[Electronic resource] / R. Gurbiel // Warsaw School of Economics Center of International Production Cooperation. - Warsaw, 2002.- Access mode: http:/ /www. eadi. org/ typo3/ fileadmin/ WG_Documents/Reg_WG/gurbiel.pdf.

Hasan I, Tucci C L (2010). The innovation-economic growth nexus: Global evidence. Research Policy, 39(10), 1264-1276.

Josheski D, Koteski C, (2001), The causal relationship between patent growth and growth of GDP with quarterly data in the G7 countries: cointegration, ARDL and error correction models, Munich Personal RePEc Archive (MPRA) Paper No. 33153, posted 04 Sep 2011 02:08 UTC,

Katz J S (2006). Indicators for complex innovation systems. Research Policy, 35, 893-909.

Kim Y K, Lee K, Park W G, Choo K (2012). Appropriate intellectual property protection and economic growth in countries at different levels of development.

Research Policy, 41(2), 358-375.

Maskus K, (2000). Intellectual Property Rights in the Global Economy. Washington, D.C.: Institute of International Economics. 
Pesaran M H, Shin Y, Smith R J (2001). Bounds testing approaches to the analysis of level relationships. Journal of applied econometrics, 16(3), 289-326.

Pesaran M H, Shin Y, Smith R J (2001). Bounds Testing Approaches to the Analysis of Level Relationships. J. Appl. Econometr. 16, 289-326.

Sinha D (2007). "Patents, Innovations and Economic Growth in Japan and South Korea: Evidence from individual country and panel data", Munich

Personal RePEc Archive, 2007, paper No. 2547.

Schumpeter, J.A. (1947) Capitalism, Socialism and Democracy. Harper \& Brothers Publishers, New York.

Wang Ch, (2013). The long-run effect of innovation on economic growth [Electronic resource]/ Ch.Wang.-Australia:School of Economics, 2013- Accessmode: http:// www. murdoch. edu.au/School-of-Business-and-Governance/_document/AustralianConference-of-Economists/ Thelong-run-effect-of-innovation-on-economic-growth.pdf. 